\title{
Estrategias para la medición y el manejo de la sobreocupación de los servicios de urgencias de adultos en instituciones de alta complejidad con altos volúmenes de consulta. Revisión de la literatura
}

\author{
Andrés Felipe Estrada-Atehortúa', Mateo Zuluaga-Gómez²
}

\section{RESUMEN}

La sobreocupación de los servicios de urgencias es un problema global que cada vez afecta más las instituciones de salud que atienden pacientes de mediana y alta complejidad, haciendo que estos permanezcan más tiempo en una sala de espera con la consiguiente demora en los tiempos de atención, bajo nivel de satisfacción de los usuarios, retraso en la toma de ayudas diagnósticas, retrasos al definir altas del servicio y favorecimiento de complicaciones médicas, entre otros. Para mejorar esta situación se han desarrollado estrategias como la creación de unidades de observación, unidades fast track o asionnación de citas prioritarias para los pacientes que no requieren una atención urogente, de modo adicional el triaje, los exámenes point of care y la vinculación de especialistas en medicina de urgencias. Tođo esto con el fin de mejorar la calidad de la atención de los pacientes, evitar que se presenten eventos adversos durante su proceso y disminuir la sobreocupación del servicio.

\section{PALABRAS CLAVE}

Atención en Urogencias; Medicina de Uroencias; Triaje

\footnotetext{
1 Médico especialista en Medicina de Urgencias; especialista en Gerencia de IPS. Urgentólogo, Hospital Pablo Tobón Uribe. Docente de la Facultad de Medicina, Universidad Pontificia Bolivariana. Docente adscrito Universidad CES. Línea de Investigación Medicina de Urgencias y Toxicología CES. Medellín, Colombia.

2 Médico residente de la especialización en Medicina de Urgencias, Universidad CES. Línea de Investigación Medicina de Urgencias y Toxicología CES. Médico general, Universidad Pontificia Bolivariana. Medellín, Colombia.
}

Correspondencia: Andrés Felipe Estrada-Atehortúa; andresestrada23@hotmail.com

Recibido: febrero 19 de 2019

Aceptado: junio 6 de 2019

Cómo citar: Estrada-Atehortúa AF, Zuluaga-Gómez M. Estrategias para la medición y el manejo de la sobreocupación de los servicios de urgencias de adultos en instituciones de alta complejidad con altos volúmenes de consulta. Revisión de la literatura. latreia. 2020 Ene-Mar;33(1):68-77. D0I 10.17533/udea.iatreia.34. 


\section{SUMMARY}

Strategies for the measurement and management of overcrowding in the adult emergency services of high complexity and high consultation volumes hospitals. A literature review

The over-occupation of emergency services is a global problem that has worsened in recent years. In the health institutions that serve patients of medium and high complexity, the attention of the patients is delayed and they can stay longer in a waiting room presenting longer attention times, a low level of satisfaction of the patients and users, delay in taking diagnostic aids and in defining discharge, medical complications and progression of their diseases, among others. To improve this situation, strategies have been developed such as the creation of observation units, fast track units or assignment of priority appointments for patients who do not require urgent attention, triage, point-of-care examinations and the involvement of specialists in emergency medicine. All of this in order to improve the quality of patient care, avoid adverse events during their process and reduce the over-occupation of the service.

\section{KEYWORDS}

Emeroency Care; Emeroency Medicine; Triage

\section{INTRODUCCIÓN}

Al hacer referencia al tema de la sobreocupación de los servicios de urgencias se debe tener claro qué es el triaje, herramienta utilizada en los servicios de urgencias para priorizar la atención de los pacientes, quienes una vez son clasificados se asiona un orden de atención. A partir de la clasificación del triaje, ingresan directamente al servicio los pacientes que tienen una condición grave de salud que amenaza la vida, y se da espacio para que esperen en la sala los pacientes que necesitan atención, pero cuya conđición clínica no amenaza la vida en los siguientes minutos ${ }^{(1)}$.

No es secreto que cada día se vive una situación estresante en los servicios de urgencias debido a su sobreocupación ${ }^{(2)}$, esto sucede cuando la demanda de los servicios sobrepasa su capacidad de atención en un periodo de tiempo determinado ${ }^{(3)}$. Aun teniendo este concepto claro, ha sido difícil encontrar el punto de partida para su definición ${ }^{(4)}$.

Es difícil encontrar datos exactos en la literatura cuando se intenta de definir el alto volumen de las consultas por urgencias, sin embargo, Wang et al., encontraron que este número oscila entre 40.000 y 83.000 consultas anuales por urgencias ${ }^{(5)}$. A lo anterior, se le debe sumar el fenómeno del aumento exponencial de las consultas en los servicios de atención de urgencias en todo el mundo ${ }^{(6-8)}$.

Según el Informe Nacional de Calidad de la Atención en Salud 2015 de Colombia, el tiempo de espera para la atención de consulta en uroencias que reportaron las Instituciones Prestadoras de Servicios de Salud (IPS) en el periodo 2009-2014 incrementó de 28,71 a 32,61 minutos, como consolidado para todos los pacientes que ingresaron por urgencias, independiente de su clasificación en el triaje; incluso reportan que en el año 2014 el servicio de urgencias fue el peor calificado por los usuarios de los servicios de salud, con un indicador del 65 \% (casi una tercera parte de los usuarios inconformes) ${ }^{(9)}$. Hecho que afecta el proceso de atención a los pacientes, quienes en consecuencia, pueden optar por el abandono de las salas de uronencias ${ }^{(6,9-11)}$.

O'Connor, et al., demostraron en dos hospitales urbanos de Ottawa, Canadá que la sobreocupación de los servicios de urgencias en 1,5 reces su capacidad instalada influenciaba la clasificación del triaje y la disposición de los pacientes dentro del mismo, hasta llegar a prolongar incluso los tiempos de inicio de la atención ${ }^{(12)}$. Pines en Pensilvania, Estados Unidos, encontró que la sobreocupación de los servicios de urgencias pueden asociarse con un aumento de la frecuencia de los eventos adversos cardiovasculares, relacionados o no con un evento coronario agudo en los pacientes que esperan ser atendidos en estas instituciones ${ }^{(13)}$, incluso con un aumento en la mortalidad, retrasos en el inicio de maniobras de reanimación, en el inicio del tratamiento antibiótico en los pacientes sépticos y con un menor nivel de satisfacción de los usuarios de los servicios de urgencias. Se ha hablado de una pobre satisfacción laboral por parte de todo el personal que trabaja en los servicios de urgencias, con un deterioro demostrado de su calidad de vida por altos niveles de estrés ${ }^{(2,14-17)}$. 
De acuerdo con lo anterior, se hace necesario encontrar herramientas que ayuden a afrontar este problema y generar soluciones ${ }^{(17)}$.

\section{SOBREOCUPACIÓN EN URGENCIAS: DEFINICIONES Y CAUSAS}

Se habla de sobreocupación cuando la demanda de los servicios de urgencias sobrepasa su capacidad de atención en un periodo de tiempo determinado. Otras formas de aproximarse a la definición actual de la sobreocupación de un servicio de urgencias es cuando las visitas sobrepasan el promedio diario para la institución ${ }^{(7)} \mathrm{O}$, cuando hay una ocupación de camas en el servicio de urgencias por más de ocho horas al día en más del $90 \%$ del servicio ${ }^{(18,19)}$

Se han descrito varias causas de la sobreocupación en los servicios de urgencias; Asplin et al., con su modelo conceptual proponen tres puntos clave en la movilización de los pacientes en un servicio de urogencias ${ }^{(20)}$ :

Input: en español entrada o ingreso, consiste en la atención que busca el paciente en urogencias; en este se encuentran quienes consultan por motivos que no son una real urgencia y que podrían ser manejados en el ambiente ambulatorio. En realidad esta no es una razón para considerar sobreocupación de los servicios de urgencias, dado que no se puede controlar de forma objetiva el ingreso de los pacientes, pero es posible manejarlo de forma ágil aquellos que no requieren atención urgente con altas de consultorio y entrega de órdenes ambulatorias ${ }^{(20)}$.

Throughput: en español a lo largo de - durante el paso por urgencias. Consiste en los recursos y cuidados que se prestan en el paciente que se encuentra en urgencias ${ }^{(20)}$.

Output: en español, egreso desde el servicio de urgencias. Consiste en la movilización de pacientes desde el servicio de urgencias hacia otras dependencias incluyendo el domicilio. Este es uno de los principales problemas que se considera como la causa de la sobreocupación, dado que pone en pausa el flujo de pacientes generando acumulación en el servicio ${ }^{(20)}$.

Cuando se hace referencia a la sobreocupación de un servicio de urgencias se tiene en cuenta una relación entre la necesidad del servicio y los recursos disponibles; lo anterior es importante para entender algunas de las propuestas de medición de la sobreocupación en uronencias que se mencionan a continuación ${ }^{(11)}$.

\section{Pacientes que egresan de urgencias sin revisión médica}

Son aquellos que ingresan al servicio de urgencias ya sea porque logran ser clasificados en el triaje o no, pero que no reciben una revisión médica completa y eoresan del servicio por cualquier motivo (no desean esperar atención, fugas, etc.) (4). Se ha documentado que la presencia de especialistas en medicina de urgencias mejora estadísticamente el indicador de la calidad de la demanda no atendida, lo que impacta positivamente el servicio ${ }^{(21)}$.

\section{Tiempos de espera para ser clasificados en triaje}

Desde que el paciente ingresa al servicio de urgencias y es clasificado en un consultorio de triaje (en la mayoría de los casos por el personal de enfermería) ${ }^{(22)}$. Algunas instituciones proponen que se debe medir este indicador buscando que se cumpla una meta de clasificación en máximo 15 minutos, al menos el 85 \% del tiempo ${ }^{(23)}$.

\section{Tiempos de espera para la atención médica}

Es el tiempo que transcurre entre el momento en que el paciente es clasificado en el triaje $y$ aquel en el que es revisado por el personal médico ${ }^{(11)}$. Según reportes del año 2015 en el Informe Nacional de Calidad en Colombia, se viene presentando un aumento creciente en el tiempo de espera de 28,71 minutos en 2009-2014 a 32,61 minutos en $2015^{(9)}$. La Resolución 5596 del año 2015 determina que la atención para los pacientes clasificados en triaje I debe ser de inmediato y para los de triaje II máximo 30 minutos. No se especifica los tiempos de atención para los pacientes de triaje III, IV y $\nabla$, que son la mayoría de las consultas en urgencias ${ }^{(24)}$.

\section{Tiempo de estancia en urgencias de los pacientes ingresados}

Consiste en el tiempo que permanece en urgencias el paciente que fue revisado por el personal médico y se definió una conducta hospitalaria ${ }^{(11)}$. 
Tiempo de estancia en urgencias de los pacientes dados de alta

Consiste en el tiempo que permanecen los pacientes en urgencias desde que fueron dados de alta hasta que salen de la institución de salud ${ }^{(11,25)}$.

De lo anterior surge la necesidad de considerar los factores que afectan directamente la sobreocupación de los servicios de urgencias. Entre los hallazgos al respecto destacan, según Arkun et al., de un hospital en New York que recibe 50.000 pacientes por urgencias al año ${ }^{(26)}$, los siguientes:

\section{La categoría de triaje asignada}

Los pacientes críticamente enfermos tenían los menores tiempos de espera ${ }^{(26)}$.

\section{La ocupación del servicio de urgencias}

En momentos en los que todo el servicio se encuentra Ileno sin camillas para ubicar pacientes, se prolongan los tiempos de atención desde la llegada de los pacientes ${ }^{(26)}$.

\section{Día de la semana en que consultan los pacientes}

Usualmente los lunes es uno de los días más congestionados en los servicios de urgencias, probablemente dado que los fines de semana no hay gran número de servicios ambulatorios abiertos ${ }^{(26)}$.

Adicionalmente, se detectan factores como la rapidez de los servicios hospitalarios de laboratorio, radiología, procedimientos, cirugía, disponibilidad de camas en hospitalización (giro de cama en salas generales, es decir, la medida del número de egresos que en promedio comprometieron el uso de cada cama disponible) y salas de cuidado crítico que pueden enlentecer la circulación de los pacientes en urgencias hacia estos sitios, en caso de que los primeros estén saturados ${ }^{(26)}$.

Dentro de los factores que pueden favorecer la sobreocupación de los servicios de urgencias se han documentado también bajos ingresos económicos en la población, no pertenecer a un sistema de seguridad social en salud y no tener acceso a los servicios de salud ambulatorios ${ }^{(27)}$. Como estrategia que podría impactar en esta causa de sobreocupación se ha propuesto la implementación de la educación de la población y el fortalecimiento de los programas de atención ambulatoria de los pacientes, las consultas por aplicaciones de celular y las líneas de atención telefónica ${ }^{(27,28)}$.

\section{ESTRATEGIAS PARA LA MEDICIÓN DE LA SOBREOCUPACIÓN EN URGENCIAS Y LA PERCEPCIÓN DEL PERSONAL DE SALUD}

Se han descrito varias herramientas para medir de manera más exacta el nivel de sobreocupación de los servicios de urgencias de mediana y alta complejidad, dentro de las cuales se describen las más relevantes ${ }^{(28-32)}$.

La escala NEDOCS, sigla de National Emergency Department Overcrowding Study, es un cuestionario desarrollado en varios hospitales de altos volúmenes de consulta por urgencias en Estados Unidos. Inicialmente se basó en 23 preguntas, finalmente se validaron las más útiles, entre las que se encuentra: total de pacientes en urgencias, total de admisiones en urgencias, número de ventiladores en urgencias, tiempo de admisión más prolongado y el tiempo de espera en sala del último paciente acostado en una camilla. Con la combinación de estas variables de forma sistematizada se arrojaron seis tipos de resultados, desde que no hay sobreocupación hasta una sobreocupación grave, con un puntaje que varía de 0 a 200. Mayor a 100 puntos es considerado como sobreocupación. Los resultados iniciales indicaron una adecuada medición de la sobreocupación en urgencias ${ }^{(29)}$ (Tabla 1).

Posteriormente, en la validación en varios hospitales, se encontró que no hubo una adecuada correlación entre su resultado y el verdadero nivel de ocupación de los hospitales de altos volúmenes de ingresos por urgencias (mayores a 100.000 pacientes por año), posiblemente en respuesta a la variabilidad de la complejidad de la atención, al personal disponible y a su preparación (residentes de varios años, nivel de formación de enfermería) entre otros ${ }^{(5)}$. Todo esto refleja la limitación en la medición exacta de la palabra sobreocupación ${ }^{(33)}$ y hace referencia a la subjetividad de esta. Su definición dependerá de muchos factores como la ubicación geográfica, población cercana, el nivel de complejidad ofrecido por la institución y la disponibilidad de recursos, por lo que se recomienda que cada institución debe tener sus propias mediciones estadísticas y conocer su funcionamiento ${ }^{(5)}$. 
En Colombia, García et al., compararon la escala objetiva NEDOCS con una medición subjetiva del personal de salud de urgencias y los niveles de sobreocupación de este servicio. Encontraron que cuando el puntaje en NEDOCS es categoría igual o mayor a 5 (gravemente congestionado y peligrosamente congestionado) no hay una conciencia del riesgo que genera la sobreocupación, incluso hay diferencia entre médicos $y$ enfermeras, siendo más concordante la percepción del grupo de las enfermeras cuando se utilizaba esta escala ${ }^{(34)}$.

Hacia el año 2002 se crea una nueva herramienta llamada EDWIN, siglas de Emergency Department Work Index, que combinaba los siguientes ítems: número de pacientes en urgencias por categoría de triaje, categoría de triaje, número de médicos por turno, número de camas de tratamiento y número de pacientes ingresados por urgencias. Los resultados se dan en tres categorías: 1. Situación manejable, 2. Situación de ocupación alta 3. Servicio sobreocupado. Estos resultados se pudieron comparar con el concepto de los médicos y las enfermeras de cómo estaba el servicio de urgencias en cuanto a ocupación, resultados que concuerdan con las mediciones de la herramienta EDWIN. Lo más importante es que pudo identificar momentos de ocupación en aumento antes que los servicios colapsaran y estuvieran sobreocupados, lo que permite tomar decisiones y medidas preventivas de forma temprana. Esto se logra porque la herramienta se puede utilizar en diferentes momentos, generando mediciones dinámicas en el tiempo ${ }^{(35,36)}$. En la literatura recomienda que estas mediciones se lleven a cabo con un intervalo aproximado de 4 horas en los servicios de urgencias $^{(37)}$ (Tabla 2).

Ahalt et aI., realizaron una comparación entre NEDOCS y EDWIN, encontrando que las dos tuvieron buen desempeño al demostrar la sobreocupación cuando ${ }^{(38)}$, ya estaba establecida en el servicio de urgencias, $\mathrm{y}$ también al predecir su ocurrencia. Un trabajo realizado por Canoa et al., en la ciudad de Bogotá, Colombia, encontró que al validar en tres instituciones de alta complejidad la escala NEDOCS se obtuvieron resultados favorables para su aplicación en nuestro medio, con una buena asociación con la percepción de sobreocupación del personal de urgencias ${ }^{(39)}$.

\section{Tabla 1. Componentes de la escala NEDOCS}

\section{NEDOCS}

1.Número de camas en el servicio de urgencias

\section{Número de camas intrahospitalarias}

3.Número total de pacientes en el servicio de urgencias

4.Número de pacientes conectados a ventilación mecánica en el servicio de urgencias

5.Pacientes con mayor tiempo de espera en el servicio de urgencias (en horas)

6. Número de pacientes admitidos en el servicio de urgencias

7.Pacientes con mayor tiempo de espera en sala de espera (en horas)

\section{INTERPRETACIÓN DE RESULTADOS}

1 - 20 puntos: no ocupado

21 - 60 puntos: ocupado

61 - 100 puntos: extremadamente ocupado, pero no sobreocupado

$101-140$ puntos: sobreocupado

$141-180$ puntos: severamente sobreocupado

181 - 200 puntos: peligrosamente sobreocupado

Los puntajes se obtienen mediante un cruce de las variables mencionadas en una herramienta electrónica. Fuente: adaptación (38)

Tabla 2. Herramienta EDWIN

\begin{tabular}{|c|}
\hline EDWIN \\
\hline 1.Número de pacientes en cada nivel de triaje \\
\hline Triaje 1: \\
\hline Triaje 2: \\
\hline Triaje 3: \\
\hline Triaje 4: \\
\hline Triage 5: \\
\hline 2.Número de médicos en el turno \\
\hline 3.Camas del servicio de urgencias \\
\hline 4.Número de pacientes admitidos a urgencias en camas \\
\hline INTERPRETACIÓN DEL RESULTADO \\
\hline 0 - 1.5: situación manejable \\
\hline Mayor a 2: servicio sobreocupado \\
\hline
\end{tabular}

Los puntajes se obtienen mediante un cruce de las variables mencionadas en una herramienta electrónica. Fuente: adaptación (38)

En Argentina, Giunta et al., aplicaron la escala NEDOCS en el servicio de urgencias de un hospital de alta complejidad. Encontraron resultados favorables 
en cuanto a su uso y la percepción diagnóstica, ya que con el análisis de sus resultados se pudieron establecer oportunidades de mejora y el desarrollo de mecanismos de intervención cuando los resultados sugerían sobreocupación del servicio ${ }^{(40)}$. Dentro de las posibles causas de la sobreocupación, además de coincidir con otros trabajos en América Latina, está la falta de camas disponibles en las salas de hospitalización que represa a los pacientes en los servicios de urgencias y empeora el problema ${ }^{(34)}$

\section{ESTRATEGIAS PARA EL MANEJO DE LA SOBREOCUPACIÓN EN URGENCIAS}

\section{Asignación de consulta prioritaria en los servicios de urgencias a pacientes que no ameritan una in- tervención o un tratamiento urgente ${ }^{(41,42)}$.}

En nuestro sistema de salud (Colombia) puede ser complicado por la distribución de los recursos; sin embargo, en Antioquia se ha ido organizando un sistema similar, la plataforma CIGA. Por medio de este recurso electrónico, cada servicio de urgencias clasifica a un paciente que puede ser manejado en la menor complejidad y puede ubicarlo en un sitio oportuno y adecuado. Es decir, un paciente clasificado en Triaje III se le asigna un lugar de consulta urgente de menor complejidad en la red de su asegurador en las siguientes 2 horas, a los de Triaje IV se les da una cita prioritaria en las siguientes 24 horas 8 a los Triaje $\nabla$ en las siguientes 72 horas. De esta forma se agiliza la distribución y organización de los pacientes en urgencias disminuyendo la sobreocupación de los servicios de alta complejidad ${ }^{(42)}$.

Los resultados arrojados para el año 2017 (1 de enero al 31 de diciembre) evidencian una asionación de cita oportuna en el menor nivel de complejidad a más de 200.000 pacientes, ahorrando al sistema de salud aproximadamente 5.000 millones de pesos (teniendo en cuenta el valor diferencial de la consulta) ${ }^{(43)}$. En Hospitales de alta complejidad como el Pablo Tobón Uribe en la ciudad de Medellín, entre enero y junio del año 2018 se asignaron 3.566 citas prioritarias por el sistema CIGA, la mayoría de ellas para pacientes clasificados como Triaje IV y $\nabla$. Aún no hay mediciones deI impacto que tiene esta asionación de citas en la congestión de este servicio de urgencias, pero puede ser el comienzo de este tipo de análisis ${ }^{(44)}$. En otras instituciones de alta complejidad las citas ambulatorias oscilan entre 560 y 1.400 mensualmente, impactando del $20-35 \%$ del volumen de pacientes de Triaje IV y V que consultan mensualmente ${ }^{(23,45)}$ (Figura 1).

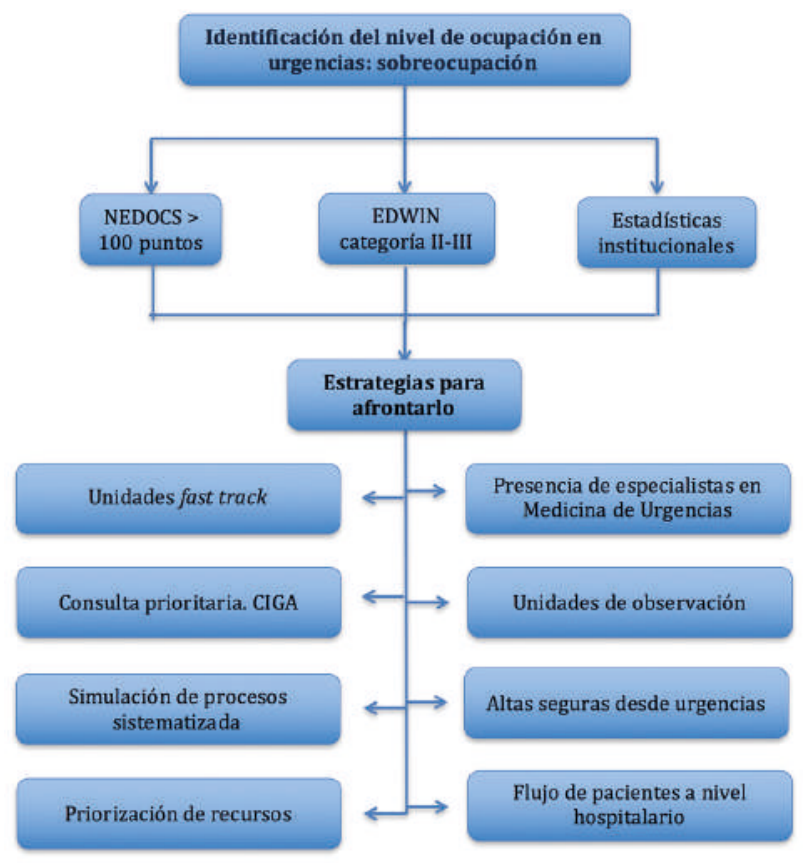

Figura 1. Cómo identificar el nivel de ocupación en urgencias y estrategias para afrontarlo Fuente: creación propia

Unidades fast track que permiten la atención rápida de pacientes no críticos que requieren administración de medicamentos o paraclínicos básicos

Esta medida ha disminuido los tiempos de espera de los pacientes críticos y tiempos de estancia en urgencias de una forma significativa ${ }^{(46)}$. Un aporte importante lo hace Garrett et al., en un estudio que comparó el flujo horizontal de los pacientes en urgencias (haciendo referencia al paciente no crítico: Triaje IV y $\nabla$ acostado en una camilla durante el proceso de su atención) contra un sistema de flujo vertical, haciendo referencia a los servicios fast track (esto es, 
pacientes ubicados en sillas y movilizados por los servicios de urgencias donde se encuentran los recursos y ayudas diagnósticas que requieren: salas de rayos $\mathrm{x}$, salas de estancia comunitarias, salas para brindar noticias y resultados de exámenes, etc.), cuyos resultados apuntan a un servicio de urgencias más eficiente con este modelo, al mejorar los tiempos de la estancia hospitalaria y el flujo de los pacientes sin comprometer la satisfacción de los usuarios ${ }^{(32)}$.

Dentro de las intervenciones que pueden mejorar los tiempos de estancia en urgencias se describen:

\section{Unidades de observación}

Es una zona de transición entre el sitio de atención inicial en urgencias y el sitio de atención efectuada en hospitalización o, el manejo ambulatorio del paciente. Se ha demostrado que estas unidades reducen los tiempos de estancia en urgencias y la sobresaturación, dado que aggilizan la administración del tratamiento para enfermedades comunes como la falla cardiaca descompensada, infección urinaria y las condiciones pulmonares crónicas que se intervienen de forma temprana y se compensan, por lo que no requieren ingreso a hospitalización, hecho que favorece altas tempranas desde los servicios de urgencias ${ }^{(36)}$.

Altas desde el servicio de urgencias: altas de consultorio ${ }^{(32)}$.

Priorización de recursos y uso de políticas hospitalarias: las pruebas en el lugar de asistencia del paciente (point-of-care: POC) son un ejemplo de esto, pues permiten tener los resultados del laboratorio por fuera de este de forma rápida, con una buena exactitud diagnóstica, ahorro de costos y agilización en la toma de decisiones clínicas de los pacientes en los servicios de urgencias ${ }^{(47)}$.

Adicionalmente, se han planteado algunas estrategias creadas para mejorar el flujo de pacientes en el servicio de urgencias y evitar la sobreocupación. Estas se basan en la optimización de los recursos existentes en una institución sin necesidad de crear nuevos espacios físicos ni generar gastos adicionales a los presupuestados por la institución, entre ellos se destacan $^{(42)}$ :

- Creación de equipos multidisciplinarios que analicen el problema al estar en el servicio de urgencias y de esta forma, poder crear estrategias para intervenir la situación puntual que esté causando problemas. Es el caso de un hospital de bajo nivel de complejidad de una ciudad en Colombia, donde se logró mejorar los tiempos de espera de los pacientes desde su ingreso a la institución, potencializando la planta del personal de la institución, mejorando el estado anímico de los trabajadores vinculándolos a la institución y redistribuyendo las asignaciones de acuerdo con las necesidades del servicio ${ }^{(48)}$.

- Creación de equipos que coordinen la asignación y el flujo de cama en todo el servicio hospitalario.

- Analizar y cruzar información estadística, por ejemplo, de los días más ocupados por análisis retrospectivos, esto para identificar la necesidad de reasignación de labores.

- Habilitación de unidades de observación en urgencias que permitan aplicar tratamientos rápidamente y dar altas tempranas.

- En momentos de sobreocupación movilizar los pacientes rápidamente a salas de observación y hospitalización, favorecer altas tempranas por parte del personal de urgencias.

Finalmente, la presencia de especialistas en urgencias ha impactado de forma positiva en múltiples ámbitos del servicio. Jui-Li et al., en una revisión retrospectiva donde midieron el impacto de la experiencia de los especialistas en medicina de urgencias en la eficiencia y en los desenlaces clínicos de los pacientes, encontraron resultados favorables al respecto. Entre más experiencia tenían los especialistas en medicina de urgencias, usualmente más de 10 años de ejercicio, se observaron menores tasas de mortalidad de los pacientes de urgencias y un menor uso de recursos en pacientes no urgentes ${ }^{(49,50)}$. Adicionalmente, la presencia de un especialista en medicina de urgencias en el servicio ayuda a optimizar el flujo de los pacientes hacia las diferentes unidades hospitalarias, definir altas tempranas de forma segura $y$ articular con otras especialidades el manejo integral de los pacientes durante su estancia en el departamento ${ }^{(51)}$, permitiendo a su vez una mayor organización y distribución del servicio cuando se compara con instituciones que no tienen 
especialistas en medicina de urgencias, lo que impacta también positivamente en la seguridad de la atención de los pacientes urgentes ${ }^{(52)}$.

\section{CONCLUSIONES}

La sobreocupación de los servicios de urgencias en el mundo y en Colombia es una realidad que todos los días genera retrasos en la atención de los pacientes críticos, con el conocido aumento de los costos en la atención médica. Existen herramientas sistematizadas como NEDOCS y EDWIN que, a través de mediciones en tiempo real, permiten dar una idea del estado de ocupación del servicio de urgencias e incluso mostrar la tendencia de la ocupación y, de esta forma, generar intervenciones que eviten llegar a una sobreocupación. Definitivamente, la presencia de especialistas en el área de medicina de urgencias tiene un impacto positivo en la oroganización del servicio, en la optimización del uso de los recursos y en la reducción de la morbimortalidad.

\section{CONFLICTOS DE INTERESES}

Ninguno por declarar.

\section{FUENTES DE FINANCIACIÓN}

Fuentes personales, no contamos con apoyo financiero para la elaboración del manuscrito.

\section{REFERENCIAS BIBLIOGRÁFICAS}

1. Wuerz RC, Milne LW, Eitel DR, Travers D, Gilboy N. Reliability and validity of a new five-level triage instrument. Acad Emerg Med. 2000;7(3):236-42.

2. Basu S, Qayyum H, Mason S. Occupational stress in the ED: a systematic literature review. Emerg Med J. 2017;34(7):441-7. DOI 10.1136/emermed-2016-205827.

3. Cano del Poso MA, Rabanaque Hernández MJ, Solana C, Martos Jiménez MC, Abad Díez MJ, Celorrio Pascual JM. Estudio de la frecuentación de un servicio de urgencias extrahospitalario. Emergencias. 2008;20:179-86.
4. McCarthy ML, Aronsky D, Jones ID, Miner JR, Band RA, Baren JM, et al. The emergency department occupancy rate: a simple measure of emergency department crowding? Ann Emerg Med. 2008;51(1):15-24.

5. Wang $H$, Robinson RD, Bunch K, Huggins CA, Watson $\mathrm{K}$, Jayswal RD, et al. The inaccuracy of determining overcrowding status by using the national ED overcrowding study tool. Am J Emerg Med. 2014;32(10):1230-6. DOI 10.1016/j.ajem.2014.07.032.

6. Hoot NR, Aronsky D. Systematic review of emeroency department crowding: causes, effects, and solutions. Ann Emerg Med. 2008;52(2):126-36. DOI 10.1016/j.annemeromed.2008.03.014.

7. Grande Ratti MF, Martínez B. Fenómeno de crowding en central de emergencias: una problemática mundial. Rev. Hosp. Ital. B.Aires. 2017;37(4).

8. Morley C, Unwin M, Peterson GM, Stankovich, J, Kinsman L. Emergency department crowding: A systematic review of causes, consequences and solutions. PLoS One. 2018;13(8):e0203316. DOI 10.1371/ journal.pone. 0203316 .

9. Colombia. Ministerio de Salud. Informe Nacional de Calidad de la Atención en Salud 2015 [Internet]. [Consultado 2018 jul 5]. Disponible en: http://cort.as/KhFQ

10. Cheng I, Lee J, Mittmann N, Tyberg J, Ramagnano $\mathrm{S}$, Kiss A, et al. Implementing wait-time reductions under Ontario government benchmarks (Pay-forResults): a Cluster Randomized Trial of the Effect of a Physician-Nurse Supplementary Triage Assistance team (MDRNSTAT) on emergency department patient wait times. BMC Emerg Med. 2013;13:17. DOI 10.1186/1471-227X-13-17.

11. Moskop JC, Sklar DP, Geiderman JM, Schears RM, Bookman KJ. Emergency Department Crowding, Part 1-Concept, Causes, and Moral Consequences. Ann Emerg Med. 2009;53(5):605-11. DOI 10.1016/j.annemergmed.2008.09.019.

12. O'Connor E, Gatien M, Weir C, Calder L. Evaluating the effect of emergency department crowding on triage destination. Int J Emerg Med. 2014;7. DOI 10.1186/1865-1380-7-16.

13. Pines JM, Pollack CV, Diercks DB, Chang AM, Shofer FS, Hollander JE. The association between emergency department crowding and adverse cardiovascular outcomes in patients with chest pain. Acad 
Emero Med. 2009;16(7):617-25. DOI 10.1111/j.15532712.2009.00456.x.

14. Rondeau KV, Francescutti LH. Emergency department overcrowding: the impact of resource scarcity on physician job satisfaction. J Healthc Manag. 2005;50(5):327-40.

15. Aranaz Andrés JM, Martínez Nogueras R, Gea Velázquez de Castro MT, Rodrigo Bartual V, Antón García P, Pajares FG. [Why do patients use hospital emeroency services on their own initiative?]. Gac Sanit. 2006;20(4):311-5.

16. Wilson W, Raj JP, Narayan G, Ghiya M, Murty S, Joseph B. Quantifying Burnout among Emergency Medicine Professionals. J Emerg Trauma Shock. 2017;10(4):199204. DOI 10.4103/JETS.JETS_36_17.

17. Schneider SM, Gallery ME, Schafermeyer R, Zwemer FL. Emergency department crowding: A point in time. Ann Emero Med. 2003;42(2):167-72.

18. Sarver JH, Cydulka RK, Baker DW. Usual source of care and nonurgent emergency department use. Acad Emerg Med. 2002;9(9):916-23.

19. Young GP, Wagner MB, Kellermann AL, Ellis J, Bouley D. Ambulatory visits to hospital emeroncy departments. Patterns and reasons for use. 24 Hours in the ED Study Group. JAMA. 1996;276(6):460-5.

20. Asplin BR, Magid DJ, Rhodes KV, Solberg LI, Lurie N, Camargo CA. A conceptual model of emergency department crowding. Ann Emerg Med. 2003;42(2):17380. DOI 10.1067/mem.2003.302.

21. Cohen Olivella E. Comparación de indicador "demanda no atendida" en urgencias, antes y después del especialista en emergencias [internet]. [Consultado 2018 jul 5]. Disponible en: http://cort.as/-KhUH

22. Abir M, Goldstick JE, Malsberger R, Williams A, Bauhoff S, Parekh $\nabla$, et al. Evaluating the impact of emergency department crowding on disposition patterns and outcomes of discharged patients. Int J Emerg Med. 2019;12(1):4. DOI 10.1186/s12245-019-0223-1.

23. Instituto Neurológico de Colombia [internet]. MedeIlín: INDEC; 2011. [Consultado 2018 sep 2]. Disponible en: http://www.institutoneurologico.org/

24. Colombia, Ministerio de Salud y Protección Social. Resolución 5596 de 2015 [internet]. [Consultado el 2018 ago 8]. Disponible en: http://cort.as/-KhZ1

25. Mullins PM, Pines JM. National ED crowding and hospital quality: results from the 2013 Hospital
Compare data. Am J Emerg Med. 2014;32(6):634-9. DOI 10.1016/j.ajem.2014.02.008.

26. Arkun A, Brigogs WM, Patel S, Datillo PA, Bove J, Birkhahn RH. Emergency Department Crowding: Factors Influencing Flow. West J Emerg Med. 2010;11(1):10.

27. Andrews H, Kass L. Non-urgent use of emergency departments: populations most likely to overestimate illness severity. Intern Emero Med. 2018;13(6). DOI 10.1007/s11739-018-1792-3.

28. Emergency Room - Detroit Medical Center | DMC | [internet]. Detroit: MI; 1994. [Consultado 2018 ago 29]. Disponible en: http://cort.as/-LAaK

29. Weiss SJ, Derlet R, Arndahl J, Ernst AA, Richards J, Fernández-Frackelton $M$, et al. Estimating the degree of emergency department overcrowding in academic medical centers: results of the National ED Overcrowding Study (NEDOCS). Acad Emerg Med. 2004;11(1):38-50.

30. Reeder TJ, Burleson DL, Garrison HG. The overcrowded emergency department: a comparison of staff perceptions. Acad Emerg Med. 2003;10(10):1059-64.

31. Raj K, Baker K, Brierley S, Murray D. National Emergency Department Overcrowding Study tool is not useful in an Australian emeronency department. Emerg Med Australas. 2006;18(3):282-8. DOI 10.1111/j.17426723.2006.00854.X.

32. Jones SS, Allen TL, Flottemesch TJ, Welch SJ. An independent evaluation of four quantitative emergency department crowding scales. Acad Emerg Med. 2006;13(11):1204-11. DOI 10.1197/j.aem.2006.05.021.

33. Hwang $\mathrm{U}$, Concato J. Care in the emergency department: how crowded is overcrowded? Acad Emerg Med. 2004;11(10):1097-101. DOI 10.1197/j. aem.2004.07.004.

34. Garcia-Romero M, Rita-Gáfaro CG, Quintero-Manzano J, Bermon A. Escala NEDOCS vs valoración subjetiva, ¿EI personal de salud en urogencias es consciente de su sobrecupo? Colomb. med. 2017;48:2.

35. Bernstein SL, Verghese V, Leung W, Lunney AT, Perez I. Development and validation of a new index to measure emergency department crowding. Acad Emerg Med. 2003;10(9):938-42.

36. Weiss SJ, Ernst AA, Nick TG. Comparison of the National Emergency Department Overcrowding Scale and the Emergency Department Work Index for 
quantifying emergency department crowding. Acad Emerg Med. 2006;13(5):513-8.

37. Wang H, Ojha RP, Robinson RD, Jackson BE, Shaikh SA, Cowden CD, et al. Optimal Measurement Interval for Emergency Department Crowding Estimation Tools. Ann Emero Med. 2017;70(5):632-9. DOI 10.1016/j.annemeromed.2017.04.012.

38. Ahalt $\nabla$, Argon NT, Ziya S, Strickler J, Mehrotra A. Comparison of emergency department crowding scores: a discrete-event simulation approach. Health Care Manag Sci. 2018;21(1):144-55. DOI 10.1007/ s10729-016-9385-z.

39. Canoa Canoa AJ, Cohen Olivella E, Lineros Montañez A, Sanchez Pedraza R. Escala NEDOCS para medir congestión en urgencias: estudio de validación en Colombia. [Especialización en Medicina de Emergencias]. Bogotá: Universidad del Rosario. InstnameUniversidad Rosario; 2010.

40. Giunta DH, Pedretti AS, Elizondo CM, Grande Ratti MF, González Bernaldo F, Waisman GD, et al. Descripción de las características del fenómeno Crowding en la Central de Emergencia de Adultos, en un hospital universitario de alta complejidad: estudio de cohorte retrospectiva. Rev. méd. Chile. 2017;145(5):557-63. DOI 10.4067/S0034-98872017000500001.

41. Stewart SW, Cheung NK, Graham CA, Rainer TH. Strategies and solutions to alleviate access block and overcrowding in emergency departments. Hong Kong Med J. 2015;21(4):345-52. DOI 10.12809/hkmj144399.

42. Moskop JC, Sklar DP, Geiderman JM, Schears RM, Bookman KJ. Emergency Department Crowding, Part 2-Barriers to Reform and Strategies to Overcome Them. Ann Emerg Med. 2009;53(5)612-7. DOI 10.1016/j.annemeromed.2008.09.024.

43. Alcaldía de Medellín [Internet]. Medellín. Alcaldía de Medellín; 1998. [Consultado 2018 ago 13]. Disponible en: http://cort.as/-5jG7.

44. Hospital Pablo Tobón Uribe [internet]. Medellín: Fecha de creación 21 de Septiembre de 2000. [Consultado el 20 de Agosto de 2018]. Disponible en: https://www. hptu.org.co/

45. Clínica Las Américas [internet]. Medellín: Clínica las Américas; 2007. [Consultado 2018 ago 30]. Disponible en: http://cort.as/-LAbs

46. Garrett JS, Berry C, Wong H, Qin H, Kline JA. The effect of vertical split-flow patient management on emergency department throughput and efficiency. Am J Emerg Med. 2018;36(9):1581-4. DOI 10.1016/j. ajem.2018.01.035.

47. Oliver Sáez P, Alonso Díaz R, Lirón Hernández J, Monzó Inglés V, Navarro Segarra X, Noval Padillo JA, et al. Guía sobre las pruebas de laboratorio en el lugar de asistencia al paciente (POCT). Rer Lab Clínico. 2016;9(2):60-80. DOI 10.1016/j.Iabcli.2016.03.003.

48. Rendón Giraldo MT, Ortega Mazo A. Diseño de un modelo para el mejoramiento del servicio de la sala de uroncias de un hospital de nivel II utilizando herramientas de simulación [Trabajo de Grado para optar el título de Ingeniero Industrial]. Zarzal: Universidad del Valle, 2013.

49. Li CJ, Syue YJ, Tsai TC, Wu KH, Lee CH, Lin YR. The Impact of Emergency Physician Seniority on Clinical Efficiency, Emergency Department Resource Use, Patient Outcomes, and Disposition Accuracy. Medicine (Baltimore). 2016;95(6):2706. DOI 10.1097/ MD. 0000000000002706 .

50. Carnevale TJ, Meng D, Wang JJ, Littlewood M. Impact of an emergency medicine decision support and risk education system on computed tomography and magnetic resonance imaging use. J Emerg Med. 2015;48(1):53-7. DOI 10.1016/j.jemermed.2014.07.033.

51. Ramlakhan S, Qayyum H, Burke D, Brown R. The safety of emergency medicine. Emerg Med J. 2016;33(4):293-9. DOI 10.1136/emermed-2014-204564.

52. Marco CA, Moskop JC, Schears RM, Stankus JL, Bookman KJ, Padela AI, et al. The ethics of health care reform: impact on emeroncy medicine. Acad Emerg Med. 2012;19(4):461-8. DOI 10.1111/j.1553-2712.2012.01313.x. 